British Journal of Mathematics \& Computer Science

9(5): 380-393, 2015, Article no.BJMCS.2015.210

ISSN : 2231-0851

SCIENCEDOMAIN international

\title{
Inconsistent Countable Set in Second Order ZFC and Nonexistence of the Strongly Inaccessible Cardinals
}

\author{
Jaykov Foukzon ${ }^{1 *}$ \\ ${ }^{1}$ Center for Mathematical Sciences, Israel Institute of Technology, Haifa, Israel.
}

Article Information

DOI: $10.9734 / B J M C S / 2015 / 16849$

Editor(s):

(1) Wei Wu, Applied Mathematics Department, Dalian University of Technology, China. (2) Sheng Zhang, Department of Mathematics, Bohai University, Jinzhou, China.

Reviewers:

(1) Anonymous, Technical School Centre of Maribor, Slovenia. (2) Anonymous, COMSATS Institute of Information Technology, Pakistan. (3) Anonymous, Kwame Nkrumah University of Science and Technology, Kumasi, Ghana.

(4) Cenap zel, AB Department of Math Bolu, Turkey. Complete Peer review History: http://www.sciencedomain.org/review-history.php?iid=1146\&id=6\&aid=9622

\section{Original Research Article}

Received: 16 February 2015

Accepted: 24 April 2015

Published: 06 June 2015

\begin{abstract}
In this article we derived an important example of the inconsistent countable set in second order $Z F C\left(Z F C_{2}\right)$ with the full second-order semantics. Main results: (i) $\neg \operatorname{Con}\left(Z F C_{2}\right)$, (ii) let $k$ be an inaccessible cardinal and $H_{k}$ is a set of all sets having hereditary size less then $k$, then $\neg \operatorname{Con}\left(Z F C+\left(V=H_{k}\right)\right)$.
\end{abstract}

Keywords: Gödel encoding; Completion of $Z \mathrm{FC}_{2}$; Russell's paradox; $\omega$-model; Henkin semantics; full second-order semantics.

\section{Introduction}

Let's remind that accordingly to naive set theory, any definable collection is a set. Let $R$ be the set of all sets that are not members of themselves. If $R$ qualifies as a member of itself, it would contradict its own definition as a set containing all sets that are not members of themselves. On the other hand, if such a set is not a member of itself, it would qualify as a member of itself by the same definition. This contradiction is Russell's paradox. In 1908, two ways of avoiding the paradox were proposed, Russell's type theory and the Zermelo set theory, the first constructed axiomatic

\footnotetext{
*Corresponding author:E-mail: jaykovfoukzon@list.ru;
} 
set theory. Zermelo's axioms went well beyond Frege's axioms of extensionality and unlimited set abstraction, and evolved into the now-canonical Zermelo-Fraenkel set theory ZFC."But how do we know that ZFC is a consistent theory, free of contradictions? The short answer is that we don't; it is a matter of faith (or of skepticism)" - E.Nelson wrote in his not published paper [1]. However, it is deemed unlikely that even $Z F C_{2}$ which is a very stronger than $Z F C$ harbors an unsuspected contradiction; it is widely believed that if $Z F C_{2}$ were inconsistent, that fact would have been uncovered by now. This much is certain $-Z F C_{2}$ is immune to the classic paradoxes of naive set theory: Russell's paradox, the Burali-Forti paradox, and Cantor's paradox.

Remark 1.1. Note that in this paper we view the second order set theory $Z F C_{2}$ under the Henkin semantics [2],[3] and under the full second-order semantics [4],[5].Thus we interpret the wff's of $Z F C_{2}$ language with the full second-order semantics as required in [4],[5].

Designation 1.1. We will be denote by $Z F C_{2}^{H s}$ set theory $Z F C_{2}$ with the Henkin semantics and we will be denote by $Z F C_{2}^{f s s}$ set theory $Z F C_{2}$ with the full second-order semantics.

Remark 1.2. There is no completeness theorem for second-order logic with the full second-order semantics. Nor do the axioms of $Z F C_{2}^{f s s}$ imply a reflection principle which ensures that if a sentence $Z$ of second-order set theory is true, then it is true in some (standard or nonstandard) model $M^{Z F C_{2}^{f s s}}$ of $Z F C_{2}^{f s s}[2]$. Let $Z$ be the conjunction of all the axioms of $Z F C_{2}^{f s s}$. We assume now that: $Z$ is true,i.e. $C o n\left(Z F C_{2}^{f s s}\right)$. It is known that the existence of a model for $Z$ requires the existence of strongly inaccessible cardinals, i.e. under $Z F C$ it can be shown that $3 b a$ is a strongly inaccessible if and only if $\left(H_{3 b a}, \in\right)$ is a model of $Z F C_{2}^{f s s}$. Thus $\neg C o n\left(Z F C_{2}^{f s s}+\right.$ $\left.\exists M^{Z F C_{2}^{f s s}}\right) \Longrightarrow \neg C o n\left(Z F C+\left(V=H_{k}\right)\right)$.In this paper we prove that $Z F C_{2}^{f s s}$ is inconsistent. We will start from a simple naive consideration.Let $\Im$ be the countable collection of all sets $X$ such that $Z F C_{2}^{f s s} \vdash \exists ! X \Psi(X)$, where $\Psi(X)$ is any 1-place open wff i.e.,

$$
\forall Y\{Y \in \Im \leftrightarrow \exists \Psi(\cdot) \exists ! X[\Psi(X) \wedge Y=X]\} .
$$

Let $X \notin_{Z F C_{2}^{f s s}} Y$ be a predicate such that $X \notin_{Z F C_{2}^{f s s}} Y \leftrightarrow Z F C_{2}^{f s s} \vdash X \notin Y$.Let $\Re$ be the countable collection of all sets such that

$$
\forall X\left[X \in \Re \leftrightarrow X \notin_{\vdash_{F C_{2}^{f}}^{f s s}} X\right] .
$$

From (1.2) one obtain

$$
\Re \in \Re \leftrightarrow \Re \notin \vdash_{Z F C_{2}^{f s s}} \Re .
$$

But obviously this is a contradiction. However contradiction (1.3) it is not a contradiction inside $Z F C_{2}^{f s s}$ for the reason that predicate $X \notin_{\vdash_{Z F C_{2}^{f s s}}} Y$ not is a predicate of $Z F C_{2}^{f s s}$ and therefore countable collections $\Im$ and $\Re$ not is a sets of $Z F C_{2}^{f s s}$. Nevertheless by using Gödel encoding the above stated contradiction can be shipped in special consistent completion of $Z F C_{2}^{f s s}$.

Remark 1.3. We note that in order to deduce $\neg \operatorname{Con}\left(Z F C_{2}^{H s}\right)$ from $\operatorname{Con}\left(Z F C_{2}^{H s}\right)$ by using Gödel encoding, one needs something more than the consistency of $Z F C_{2}^{H s}$, e.g., that $Z F C_{2}^{H s}$ has an omega-model $M_{\omega}^{Z F C_{2}^{H s}}$ or an standard model $M_{\mathrm{st}}^{Z F C_{2}^{H s}}$ i.e., a model in which the integers are the standard integers [6]. To put it another way, why should we believe a statement just because there's a $Z F C_{2}^{H s}$-proof of it? It's clear that if $Z F C_{2}^{H s}$ is inconsistent, then we won't believe $Z F C_{2}^{H s}$-proofs. What's slightly more subtle is that the mere consistency of $Z F C_{2}$ isn't quite enough to get us to believe arithmetical theorems of $Z F C_{2}^{H s}$; we must also believe that these arithmetical theorems 
are asserting something about the standard naturals. It is "conceivable" that $Z F C_{2}^{H s}$ might be consistent but that the only nonstandard models $M_{\mathrm{Nst}}^{Z F C_{2}^{H s}}$ it has are those in which the integers are nonstandard, in which case we might not "believe" an arithmetical statement such as " $Z F C_{2}^{H s}$ is inconsistent" even if there is a $Z F C_{2}^{H s}$-proof of it.

Remark 1.4. However assumption $\exists M_{\mathrm{st}}^{Z F C_{2}^{H s}}$ is not necessary. Note that in any nonstandard model $M_{\mathbf{N s t}}^{Z_{2}^{H s}}$ of the second-order arithmetic $Z_{2}^{H s}$ the terms $\overline{0}, \mathbf{S} \overline{0}=\overline{1}, \mathbf{S S} \overline{\mathbf{0}}=\overline{2}, \ldots$ comprise the initial segment isomorphic to $M_{\mathrm{st}}^{Z_{2}^{H s}} \subset M_{\mathrm{Nst}}^{Z_{2}^{H s}}$. This initial segment is called the standard cut of the $M_{\mathbf{N s t}}^{Z_{2}^{H s}}$. The order type of any nonstandard model of $M_{\mathbf{N s t}}^{Z_{2}^{H s}}$ is equal to $\mathbb{N}+A \times \mathbb{Z}$ for some linear order $A[6],[7]$. Thus one can to choose Gödel encoding inside $M_{\mathbf{s t}}^{Z_{2}^{H s}}$.

Remark 1.5. However there is no any problem as mentioned above in second order set theory $Z F C_{2}$ with the full second-order semantics becouse corresponding second order arithmetic $Z_{2}^{f s s}$ is categorical.

Remark 1.6. Note if we view second-order arithmetic $Z_{2}$ as a theory in first-order predicate calculus. Thus a model $M^{Z_{2}}$ of the language of second-order arithmetic $Z_{2}$ consists of a set $M$ (which forms the range of individual variables) together with a constant 0 (an element of $M$ ), a function $S$ from $M$ to $M$, two binary operations + and $\times$ on $M$, a binary relation $<$ on $M$, and a collection $D$ of subsets of $M$, which is the range of the set variables. When $D$ is the full powerset of $M$, the model $M^{Z_{2}}$ is called a full model. The use of full second-order semantics is equivalent to limiting the models of second-order arithmetic to the full models. In fact, the axioms of second-order arithmetic have only one full model. This follows from the fact that the axioms of Peano arithmetic with the second-order induction axiom have only one model under second-order semantics, i.e. $Z_{2}$, with the full semantics, is categorical by Dedekind's argument, so has only one model up to isomorphism. When $M$ is the usual set of natural numbers with its usual operations, $M^{Z_{2}}$ is called an omega model. In this case we may identify the model with $D$, its collection of sets of naturals, because this set is enough to completely determine an omega model. The unique full omega-model $M_{\omega}^{Z_{2}^{f s s}}$, which is the usual set of natural numbers with its usual structure and all its subsets, is called the intended or standard model of second-order arithmetic.

\section{Derivation of the Inconsistent Countable Set in $Z F C_{2}^{H s}+\exists M^{Z F C_{2}^{H s}}$}

Let $\mathbf{T h}$ be some fixed, but unspecified, consistent formal theory. For later convenience, we assume that the encoding is done in some fixed formal second order theory $\mathbf{S}$ and that $\mathbf{T h}$ contains $\mathbf{S}$.The sense in which $\mathbf{S}$ is contained in $\mathbf{T h}$ is better exemplified than explained: if $\mathbf{S}$ is a formal system of a second order arithmetic $Z_{2}^{H s}$ and $\mathbf{T h}$ is, say, $Z F C_{2}^{H s}$, then $\mathbf{T h}$ contains $\mathbf{S}$ in the sense that there is a well-known embedding, or interpretation, of $\mathbf{S}$ in $\mathbf{T h}$. Since encoding is to take place in $\mathbf{S}$, it will have to have a large supply of constants and closed terms to be used as codes. (e.g. in formal arithmetic, one has $\overline{0}, \overline{1}, \ldots$.) $\mathbf{S}$ will also have certain function symbols to be described shortly.To each formula, $\Phi$, of the language of $\mathbf{T h}$ is assigned a closed term, $[\Phi]^{c}$, called the code of $\Phi$. We note that if $\Phi(x)$ is a formula with free variable $x$, then $[\Phi(x)]^{c}$ is a closed term encoding the formula $\Phi(x)$ with $x$ viewed as a syntactic object and not as a parameter. Corresponding to the logical connectives and quantifiers are function symbols, neg $(\cdot), i m p(\cdot)$, etc., such that, for all formulae $\Phi, \Psi: \mathbf{S} \vdash \operatorname{neg}\left([\Phi]^{c}\right)=[\neg \Phi]^{c}, \mathbf{S} \vdash \operatorname{imp}\left([\Phi]^{c},[\Psi]^{c}\right)=[\Phi \rightarrow \Psi]^{c}$ etc. Of particular importance is the substitution operator, represented by the function symbol $s u b(\cdot, \cdot)$. For formulae $\Phi(x)$, terms $t$ with codes $[t]^{c}$ : 


$$
\mathbf{S} \vdash \operatorname{sub}\left([\Phi(x)]^{c},[t]^{c}\right)=[\Phi(t)]^{c} .
$$

It well known [8] that one can also encode derivations and have a binary relation $\operatorname{Prov}_{\mathbf{T h}}(x, y)$ (read " $x$ proves $y$ " or " $x$ is a proof of $y ")$ such that for closed $t_{1}, t_{2}: \mathbf{S} \vdash \operatorname{Prov}_{\mathbf{T h}}\left(t_{1}, t_{2}\right)$ iff $t_{1}$ is the code of a derivation in $\mathbf{T h}$ of the formula with code $t_{2}$. It follows that

$$
\mathbf{T h} \vdash \Phi \text { iff } \mathbf{S} \vdash \operatorname{Prov}_{\mathbf{T h}}\left(t,[\Phi]^{c}\right)
$$

for some closed term $t$.Thus one can define

$$
\operatorname{Pr}_{\mathbf{T h}}(y) \leftrightarrow \exists x \operatorname{Prov}_{\mathbf{T h}}(x, y),
$$

and therefore one obtain a predicate asserting provability. We note that is not always the case that [8]:

$$
\mathbf{T h} \vdash \Phi \text { iff } \mathbf{S} \vdash \mathbf{P r}_{\mathbf{T h}}\left([\Phi]^{c}\right) .
$$

It well known [8] that the above encoding can be carried out in such a way that the following important conditions D1, D2 and D3 are meet for all sentences [8]:

$$
\begin{gathered}
\text { D1. Th } \vdash \Phi \text { implies } \mathbf{S} \vdash \mathbf{P r}_{\mathbf{T h}}\left([\Phi]^{c}\right), \\
\text { D2.S } \vdash \mathbf{P r}_{\mathbf{T h}}\left([\Phi]^{c}\right) \rightarrow \mathbf{P r}_{\mathbf{T h}}\left(\left[\mathbf{P r}_{\mathbf{T h}}\left([\Phi]^{c}\right)\right]^{c}\right), \\
\text { D3.S } \vdash \mathbf{P r}_{\mathbf{T h}}\left([\Phi]^{c}\right) \wedge \mathbf{P r}_{\mathbf{T h}}\left([\Phi \rightarrow \Psi]^{c}\right) \rightarrow \mathbf{P r}_{\mathbf{T h}}\left([\Psi]^{c}\right) .
\end{gathered}
$$

Conditions D1, D2 and D3 are called the Derivability Conditions.

Lemma 2.1. Assume that: (i) $\operatorname{Con}(\mathbf{T h})$ and (ii) $\mathbf{T h} \vdash \mathbf{P r}_{\mathbf{T h}}\left([\Phi]^{c}\right)$, where $\Phi$ is a closed formula. Then $\mathbf{T h} \nvdash \mathbf{P r}_{\mathbf{T h}}\left([\neg \Phi]^{c}\right)$.

Proof. Let $\mathrm{Con}_{\mathrm{Th}}(\Phi)$ be a formula

$$
\left\{\begin{array}{c}
\operatorname{Con}_{\mathbf{T h}}(\Phi) \triangleq \forall t_{1} \forall t_{2} \neg\left[\operatorname{Prov}_{\mathbf{T h}}\left(t_{1},[\Phi]^{c}\right) \wedge \operatorname{Prov}_{\mathbf{T h}}\left(t_{2}, n e g\left([\Phi]^{c}\right)\right)\right] \leftrightarrow \\
\neg \exists t_{1} \neg \exists t_{2}\left[\operatorname{Prov}_{\mathbf{T h}}\left(t_{1},[\Phi]^{c}\right) \wedge \operatorname{Prov}_{\mathbf{T h}}\left(t_{2}, n e g\left([\Phi]^{c}\right)\right)\right] .
\end{array}\right.
$$

where $t_{1}, t_{2}$ is a closed term. We note that $\mathbf{T h}+\operatorname{Con}(\mathbf{T h}) \vdash \operatorname{Con}_{\mathbf{T h}}(\Phi)$ for any closed $\Phi$. Suppose that $\mathbf{T h} \vdash \mathbf{P r}_{\mathbf{T h}}\left([\neg \Phi]^{c}\right)$, then (ii) gives

$$
\mathbf{T h} \vdash \operatorname{Pr}_{\mathbf{T h}}\left([\Phi]^{c}\right) \wedge \operatorname{Pr}_{\mathbf{T h}}\left([\neg \Phi]^{c}\right) .
$$

From (2.3) and (2.7) we obtain

$$
\exists t_{1} \exists t_{2}\left[\operatorname{Prov}_{\mathbf{T h}}\left(t_{1},[\Phi]^{c}\right) \wedge \operatorname{Prov}_{\mathbf{T h}}\left(t_{2}, \operatorname{neg}\left([\Phi]^{c}\right)\right)\right] .
$$

But the formula (2.6) contradicts the formula (2.8). Therefore $\mathbf{T h} \nvdash \mathbf{P r}_{\mathbf{T h}}\left([\neg \Phi]^{c}\right)$.

Lemma 2.2. Assume that : $(i) \operatorname{Con}(\mathbf{T h})$ and (ii) $\mathbf{T h} \vdash \mathbf{P r}_{\mathbf{T h}}\left([\neg \Phi]^{c}\right)$, where $\Phi$ is a closed formula. Then $\operatorname{Th} \nvdash \operatorname{Pr}_{\mathbf{T h}}\left([\Phi]^{c}\right)$.

Assumption 2.1. Let Thbe an second order theory with the Henkin semantics. We assume now that:

(i) the language of $\mathbf{T h}$ consists of:

numerals $\overline{0}, \overline{1}, \ldots$

countable set of the numerical variables: $\left\{v_{0}, v_{1}, \ldots\right\}$

countable set of the set variables: $=\{x, y, z, X, Y, Z, \Re, \ldots\}$

countable set of the $n$-ary function symbols: $f_{0}^{n}, f_{1}^{n}, \ldots$

countable set of the $n$-ary relation symbols: $R_{0}^{n}, R_{1}^{n}, \ldots$ 
connectives: $\neg, \rightarrow$

quantifier: $\forall$.

(ii) Th contains $Z F C_{2}$,

(iii) Th has an an $\omega$-model $M_{\omega}^{\mathbf{T h}}$ or

(iv) Th has an nonstandard model $M_{N s t .}^{\mathrm{Th}}$.

Definition 2.1. An Th-wff $\Phi$ (well-formed formula $\Phi$ ) is closed - i.e. $\Phi$ is a sentence - if it has no free variables; a wff is open if it has free variables. We'll use the slang ' $k$-place open wff' to mean a wff with $k$ distinct free variables.

Definition 2.2. We said that, $\mathbf{T h}^{\#}$ is a nice theory or a nice extension of the $\mathbf{T h}$ iff

(i) $\mathbf{T h}^{\#}$ contains $\mathbf{T h}$;(ii) Let $\Phi$ be any closed formula, then $\mathbf{T h} \vdash \mathbf{P r}_{\mathbf{T h}}\left([\Phi]^{c}\right)$ implies $\mathbf{T h} \mathbf{h}^{\#} \vdash \Phi$.

Definition 2.3. We said that, $\mathbf{T h} \mathbf{h}^{\#}$ is a maximally nice theory or a maximally nice extension of the $\mathbf{T h}$ iff $\mathbf{T h} \mathbf{h}^{\#}$ is consistent and for any consistent nice extension $\mathbf{T} \mathbf{h}^{\prime}$ of the $\mathbf{T h}: \mathbf{D e d}\left(\mathbf{T h} \mathbf{h}^{\#}\right) \subseteq$ $\operatorname{Ded}\left(\mathbf{T h}^{\prime}\right)$ implies $\operatorname{Ded}\left(\mathbf{T h} \mathbf{h}^{\#}\right)=\operatorname{Ded}\left(\mathbf{T h}^{\prime}\right)$.

Remark 2.1. We note that a theory $\mathbf{T h}{ }^{\#}$ depend on model $M_{\omega}^{\mathbf{T h}}$ or $M_{N s t}^{\mathbf{T h}}$, i.e. $\mathbf{T} \mathbf{T h}^{\#}=$ $\mathbf{T h}^{\#}\left[M_{\omega}^{\mathbf{T h}}\right]$ or $\mathbf{T h}^{\#}=\mathbf{T h}{ }^{\#}\left[M_{\mathbf{N s t}}^{\mathbf{T h}}\right]$ correspondingly. We will consider the case $\mathbf{T h}^{\#} \triangleq \mathbf{T h} \#\left[M_{\omega}^{\mathbf{T h}}\right]$ without loss of generality.

Proposition 2.1. Assume that (i) Con (Th) and (ii) Th has an $\omega$-model $M_{\omega}^{\mathbf{T h}}$. Then theory $\mathbf{T h}$ can be extended to a maximally consistent nice theory $\mathbf{T h}^{\#} \triangleq \mathbf{T h}^{\#}\left[M_{\omega}^{\mathbf{T h}}\right]$.

Proof. $\operatorname{Let} \Phi_{1} \ldots \Phi_{i} \ldots$ be an enumeration of all wff's of the theory $\mathbf{T h}$ (this can be achieved if the set of propositional variables can be enumerated). Define a chain $\wp=\left\{\mathbf{T h}_{i} \mid i \in \mathbb{N}\right\}, \mathbf{T h} \mathbf{h}_{1}=\mathbf{T h}$ of consistent theories inductively as follows: assume that theory $\mathbf{T h}_{i}$ is defined. (i) Suppose that a statement (2.9) is satisfied

$$
\mathbf{T h}_{i} \vdash \mathbf{P r}_{\mathbf{T h}_{i}}\left(\left[\Phi_{i}\right]^{c}\right) \text { and }\left[\mathbf{T h}_{i} \nvdash \Phi_{i}\right] \wedge\left[M_{\omega}^{\mathbf{T h}} \models \Phi_{i}\right] .
$$

Then we define a theory $\mathbf{T} \mathbf{h}_{i+1}$ as follows $\mathbf{T h}_{i+1} \triangleq \mathbf{T} \mathbf{h}_{i} \cup\left\{\Phi_{i}\right\}$.Using Lemma 2.1 we will rewrite the condition (2.9) symbolically as follows

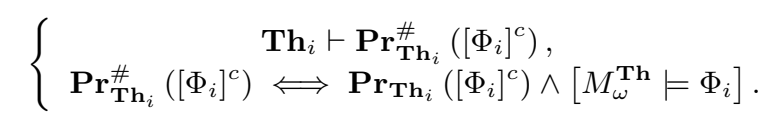

(ii) Suppose that a statement (2.11) is satisfied

$$
\mathbf{T h}_{i} \vdash \mathbf{P r}_{\mathbf{T h}_{i}}\left(\left[\neg \Phi_{i}\right]^{c}\right) \text { and }\left[\mathbf{T h}_{i} \nvdash \neg \Phi_{i}\right] \wedge\left[M_{\omega}^{\mathbf{T h}} \models \neg \Phi_{i}\right] .
$$

Then we define theory $\mathbf{T} \mathbf{h}_{i+1}$ as follows: $\mathbf{T} \mathbf{h}_{i+1} \triangleq \mathbf{T} \mathbf{h}_{i} \cup\left\{\neg \Phi_{i}\right\}$.

Using Lemma 2.2 we will rewrite the condition (2.11) symbolically as follows

$$
\left\{\begin{array}{c}
\mathbf{T h}_{i} \vdash \mathbf{P r}_{\mathbf{T h}_{i}}^{\#}\left(\left[\neg \Phi_{i}\right]^{c}\right), \\
\mathbf{P r}_{\mathbf{T h}_{i}}^{\#}\left(\left[\neg \Phi_{i}\right]^{c}\right) \Longleftrightarrow \mathbf{P r}_{\mathbf{T h}_{i}}\left(\left[\neg \Phi_{i}\right]^{c}\right) \wedge\left[M_{\omega}^{\mathbf{T h}} \models \neg \Phi_{i}\right] .
\end{array}\right.
$$

(iii) Suppose that a statement (2.13) is satisfied

$$
\mathbf{T h}_{i} \vdash \mathbf{P r}_{\mathbf{T h}_{i}}\left(\left[\Phi_{i}\right]^{c}\right) \text { and } \mathbf{T h}_{i} \vdash \mathbf{P r}_{\mathbf{T h}}\left(\left[\Phi_{i}\right]^{c}\right) \Longrightarrow \Phi_{i} .
$$

We will rewrite the condition (2.13) symbolically as follows 


$$
\left\{\begin{array}{c}
\operatorname{Th}_{i} \vdash \operatorname{Pr}_{\mathbf{T h}_{i}}^{*}\left(\left[\Phi_{i}\right]^{c}\right) \\
\operatorname{Pr}_{\mathbf{T h}_{i}}^{*}\left(\left[\Phi_{i}\right]^{c}\right) \Longleftrightarrow \operatorname{Pr}_{\operatorname{Th}_{i}}\left(\left[\Phi_{i}\right]^{c}\right) \wedge\left[\operatorname{Pr}_{\mathbf{T h}_{i}}\left(\left[\Phi_{i}\right]^{c}\right) \Longrightarrow \Phi_{i}\right]
\end{array}\right.
$$

Then we define a theory $\mathbf{T} \mathbf{h}_{i+1}$ as follows: $\mathbf{T} \mathbf{h}_{i+1} \triangleq \mathbf{T} \mathbf{h}_{i}$.

(iv) Suppose that a statement (2.15) is satisfied

$$
\mathbf{T h}_{i} \vdash \mathbf{P r}_{\mathbf{T h}_{i}}\left(\left[\neg \Phi_{i}\right]^{c}\right) \text { and } \mathbf{T h}_{i} \vdash \mathbf{P r}_{\mathbf{T h}_{i}}\left(\left[\neg \Phi_{i}\right]^{c}\right) \Longrightarrow \neg \Phi_{i} .
$$

We will rewrite the condition (2.15) symbolically as follows

$$
\left\{\begin{array}{c}
\operatorname{Th}_{i} \vdash \mathbf{P r}_{\mathbf{T h}_{i}}^{*}\left(\left[\Phi_{i}\right]^{c}\right) \\
\operatorname{Pr}_{\mathbf{T h}_{i}}^{*}\left(\left[\neg \Phi_{i}\right]^{c}\right) \Longleftrightarrow \operatorname{Pr}_{\mathbf{T h}_{i}}\left(\left[\neg \Phi_{i}\right]^{c}\right) \wedge\left[\mathbf{P r}_{\mathbf{T h}}\left(\left[\neg \Phi_{i}\right]^{c}\right) \Longrightarrow \neg \Phi_{i}\right]
\end{array}\right.
$$

Then we define a theory $\mathbf{T} \mathbf{h}_{i+1}$ as follows: $\mathbf{T} \mathbf{h}_{i+1} \triangleq \mathbf{T} \mathbf{h}_{i}$. We define now a theory $\mathbf{T} \mathbf{h}^{\#}$ as follows:

$$
\mathbf{T h}^{\#} \triangleq \bigcup_{i \in \mathbb{N}} \mathbf{T h}_{i}
$$

First, notice that each $\mathbf{T} \mathbf{h}_{i}$ is consistent. This is done by induction on $i$ and by Lemmas 2.1-2.2. By assumption, the case is true when $i=1$.Now, suppose $\mathbf{T} \mathbf{h}_{i}$ is consistent. Then its deductive closure $\operatorname{Ded}\left(\mathbf{T h}_{i}\right)$ is also consistent. If a statement (2.14) is satisfied,i.e. $\mathbf{T h} \vdash \mathbf{P r}_{\mathbf{T h}}\left(\left[\Phi_{i}\right]^{c}\right)$ and $\mathbf{T h} \vdash \Phi_{i}$, then clearly $\mathbf{T} \mathbf{h}_{i+1} \triangleq \mathbf{T} \mathbf{h}_{i} \cup\left\{\Phi_{i}\right\}$ is consistent since it is a subset of closure $\operatorname{Ded}\left(\mathbf{T} \mathbf{h}_{i}\right)$.If a statement (2.15) is satisfied,i.e. $\mathbf{T h} \vdash \mathbf{P r}_{\mathbf{T h}}\left(\left[\neg \Phi_{i}\right]^{c}\right)$ and $\mathbf{T h} \vdash \neg \Phi_{i}$, then clearly $\mathbf{T h}_{i+1} \triangleq \mathbf{T h}_{i} \cup\left\{\neg \Phi_{i}\right\}$ is consistent since it is a subset of closure $\operatorname{Ded}\left(\mathbf{T} \mathbf{h}_{i}\right)$. Otherwise:(i) if a statement (2.9) is satisfied,i.e. $\mathbf{T h} \vdash \mathbf{P r}_{\mathbf{T h}}\left(\left[\Phi_{i}\right]^{c}\right)$ and $\left[\mathbf{T h}_{i} \nvdash \Phi_{i}\right] \wedge\left[M_{\omega}^{\mathbf{T h}} \models \Phi_{i}\right]$ then clearly $\mathbf{T h}_{i+1} \triangleq \mathbf{T h}_{i} \cup\left\{\Phi_{i}\right\}$ is consistent by Lemma 2.1 and by one of the standard properties of consistency: $\Delta \cup\{A\}$ is consistent iff $\Delta \nvdash \neg A$; (ii) if a statement (2.11) is satisfied,i.e. $\mathbf{T h}_{i} \vdash \mathbf{P r}_{\mathbf{T h}_{i}}\left(\left[\neg \Phi_{i}\right]^{c}\right)$ and $\left[\mathbf{T h}_{i} \nvdash \neg \Phi_{i}\right] \wedge\left[M_{\omega}^{\mathbf{T h}} \models \neg \Phi_{i}\right]$ then clearly $\mathbf{T h}_{i+1} \triangleq \mathbf{T} \mathbf{h}_{i} \cup\left\{\neg \Phi_{i}\right\}$ is consistent by Lemma 2.2 and by one of the standard properties of consistency: $\Delta \cup\{\neg A\}$ is consistent iff $\Delta \nvdash A$.Next, notice Ded $\left(\mathbf{T h}{ }^{\#}\right)$ is maximally consistent nice extension of the Ded (Th) .Ded $\left(\mathbf{T h}{ }^{\#}\right)$ is consistent because, by the standard Lemma 2.3 belov, it is the union of a chain of consistent sets. To see that Ded $\left(\mathbf{T h} \mathbf{h}^{\#}\right)$ is maximal, pick any wff $\Phi$. Then $\Phi$ is some $\Phi_{i}$ in the enumerated list of all wff's. Therefore for any $\Phi$ such that $\mathbf{T h}_{i} \vdash \mathbf{P r}_{\mathbf{T h}}\left([\Phi]^{c}\right)$ or $\mathbf{T h}_{i} \vdash \mathbf{P r}_{\mathbf{T h}_{i}}\left([\neg \Phi]^{c}\right)$, either $\Phi \in \mathbf{T h} \mathbf{h}^{\#}$ or $\neg \Phi \in \mathbf{T} \mathbf{h}^{\#}$. Since Ded $\left(\mathbf{T} \mathbf{h}_{i+1}\right) \subseteq \operatorname{Ded}\left(\mathbf{T h} \mathbf{h}^{\#}\right)$, we have $\Phi \in \operatorname{Ded}\left(\mathbf{T h} \mathbf{h}^{\#}\right)$ or $\neg \Phi \in \operatorname{Ded}\left(\mathbf{T h} \mathbf{h}^{\#}\right)$, which implies that $\mathbf{D e d}\left(\mathbf{T} \mathbf{h}^{\#}\right)$ is maximally consistent nice extension of the Ded (Th).

Lemma 2.3. The union of $a$ chain $\wp=\left\{\boldsymbol{\Gamma}_{i} \mid i \in \mathbb{N}\right\}$ of consistent sets $\boldsymbol{\Gamma}_{i}$, ordered by $\subseteq$, is consistent.

Definition 2.4. We define now predicate $\mathbf{P r}_{\mathbf{T h}}\left(\left[\Phi_{i}\right]^{c}\right)$ asserting provability in $\mathbf{T h} \#$ :

$$
\left\{\begin{aligned}
\mathbf{P r}_{\mathbf{T h} \#}\left(\left[\Phi_{i}\right]^{c}\right) & \Longleftrightarrow\left[\mathbf{P r}_{\mathbf{T h}_{i}}^{\#}\left(\left[\Phi_{i}\right]^{c}\right)\right] \vee\left[\mathbf{P r}_{\mathbf{T} \mathbf{h}_{i}}^{*}\left(\left[\Phi_{i}\right]^{c}\right)\right], \\
\mathbf{P r}_{\mathbf{T h} \#}\left(\left[\neg \Phi_{i}\right]^{c}\right) & \Longleftrightarrow\left[\mathbf{P r}_{\mathbf{T h}_{i}}^{\#}\left(\left[\neg \Phi_{i}\right]^{c}\right)\right] \vee\left[\mathbf{P r}_{\mathbf{T h}}^{*}\left(\left[\neg \Phi_{i}\right]^{c}\right)\right] .
\end{aligned}\right.
$$

Definition 2.5. Let $\Psi=\Psi(x)$ be one-place open wff such that the conditions:

(*) $\mathbf{T h} \vdash \exists ! x_{\Psi}\left[\Psi\left(x_{\Psi}\right)\right]$ or

$(* *) \mathbf{T h} \vdash \mathbf{P r}_{\mathbf{T h}}\left(\left[\exists ! x_{\Psi}\left[\Psi\left(x_{\Psi}\right)\right]\right]^{c}\right)$ and $M_{\omega}^{\mathbf{T h}} \models \exists ! x_{\Psi}\left[\Psi\left(x_{\Psi}\right)\right]$ is satisfied.

Then we said that, a set $y$ is a $\mathbf{T} \mathbf{h}^{\#}$-set iff there is exist one-place open wff $\Psi(x)$ such that $y=x_{\Psi}$. We write $y\left[\mathbf{T h}^{\#}\right]$ iff $y$ is a $\mathbf{T h}^{\#}$-set. Remark 2.2. Note that $[(*) \vee(* *)] \Longrightarrow \mathbf{T h}^{\#} \vdash$ $\exists ! x_{\Psi}\left[\Psi\left(x_{\Psi}\right)\right]$. 
Remark 2.3. Note that $y\left[\mathbf{T h}^{\#}\right] \Longleftrightarrow \exists \Psi\left[\left(y=x_{\Psi}\right) \wedge \mathbf{P r}_{\mathbf{T h}} \#\left(\left[\exists ! x_{\Psi}\left[\Psi\left(x_{\Psi}\right)\right]\right]^{c}\right)\right]$

Definition 2.6. Let $\Im$ be a collection such that : $\forall x\left[x \in \Im \leftrightarrow x\right.$ is a $\mathbf{T h}^{\#}$-set $]$.

Proposition 2.2. Collection $\Im$ is a $\mathbf{T h}^{\#}{ }_{\text {-set. }}$

Proof. Let us consider an one-place open wff $\Psi(x)$ such that conditions $(*)$ or $(* *)$ is satisfied, i.e. $\mathbf{T h}^{\#} \vdash \exists ! x_{\Psi}\left[\Psi\left(x_{\Psi}\right)\right]$. We note that there exists countable collection $\Psi$ of the one-place open wff's $\Psi=\left\{\Psi_{n}(x)\right\}_{n \in \mathbb{N}}$ such that: (i) $\Psi(x) \in_{\Psi}$ and (ii)

$$
\left\{\begin{aligned}
\mathbf{T h} \vdash \exists ! x_{\Psi}\left[\left[\Psi\left(x_{\Psi}\right)\right]\right. & \left.\wedge\left\{\forall n(n \in \mathbb{N})\left[\Psi\left(x_{\Psi}\right) \leftrightarrow \Psi_{n}\left(x_{\Psi}\right)\right]\right\}\right] \\
\mathbf{T h} \vdash \exists ! x_{\Psi}\left[\operatorname{Pr}_{\mathbf{T h}}\left(\left[\Psi\left(x_{\Psi}\right)\right]^{c}\right)\right. & \left.\wedge\left\{\forall n(n \in \mathbb{N}) \operatorname{Pr}_{\mathbf{T h}}\left(\left[\Psi\left(x_{\Psi}\right) \leftrightarrow \Psi_{n}\left(x_{\Psi}\right)\right]^{c}\right)\right\}\right] \\
M_{\omega}^{\mathbf{T h}} \models \exists ! x_{\Psi}\left[\left[\Psi\left(x_{\Psi}\right)\right]\right. & \left.\wedge\left\{\forall n(n \in \mathbb{N})\left[\Psi\left(x_{\Psi}\right) \leftrightarrow \Psi_{n}\left(x_{\Psi}\right)\right]\right\}\right]
\end{aligned}\right.
$$

or of the equivalent form

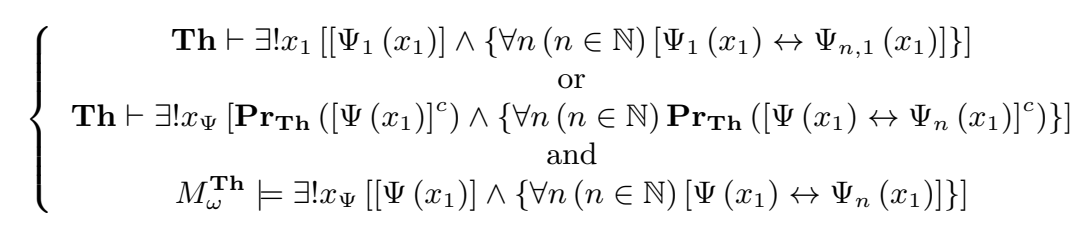

where we set $\Psi(x)=\Psi_{1}\left(x_{1}\right), \Psi_{n}\left(x_{1}\right)=\Psi_{n, 1}\left(x_{1}\right)$ and $x_{\Psi}=x_{1}$. We note that any collection $\Psi_{k}=\left\{\Psi_{n, k}(x)\right\}_{n \in \mathbb{N}}, k=1,2, \ldots$ such above defines an unique set $x_{\Psi_{k}}$, i.e. $\quad \Psi_{k_{1}} \bigcap_{\Psi_{k_{2}}}=\varnothing$ iff $x_{\Psi_{k_{1}}} \neq x_{\Psi_{k_{2}}}$. We note that collections $\Psi_{k}, k=1,2, .$. is no part of the $Z F C_{2}$,i.e. collection $\Psi_{k}$ there is no set in sense of $Z F C_{2}$. However that is no problem, because by using Gödel numbering one can to replace any collection $\Psi_{k}, k=1,2, .$. by collection $\Theta_{k}=g\left(\Psi_{k}\right)$ of the corresponding Gödel numbers such that

$$
\Theta_{k}=g\left(\Psi_{k}\right)=\left\{g\left(\Psi_{n, k}\left(x_{k}\right)\right)\right\}_{n \in \mathbb{N}}, k=1,2, \ldots .
$$

It is easy to prove that any collection $\Theta_{k}=g\left(\Psi_{k}\right), k=1,2, .$. is a $\mathbf{T h}^{\#}$-set.This is done by Gödel encoding [8],[9] of the statament (2.19) by Proposition 2.1 and by axiom schema of separation [10]. Let $g_{n, k}=g\left(\Psi_{n, k}\left(x_{k}\right)\right), k=1,2, .$. be a Gödel number of the wff $\Psi_{n, k}\left(x_{k}\right)$. Therefore $g(k)=$ $\left\{g_{n, k}\right\}_{n \in \mathbb{N}}$, where we set ${ }_{k}=\Psi_{k}, k=1,2, .$. and

$$
\forall k_{1} \forall k_{2}\left[\left\{g_{n, k_{1}}\right\}_{n \in \mathbb{N}} \cap\left\{g_{n, k_{2}}\right\}_{n \in \mathbb{N}}=\varnothing \leftrightarrow x_{k_{1}} \neq x_{k_{2}}\right] .
$$

Let $\left\{\left\{g_{n, k}\right\}_{n \in \mathbb{N}}\right\}_{k \in \mathbb{N}}$ be a family of the all sets $\left\{g_{n, k}\right\}_{n \in \mathbb{N}}$. By axiom of choice [10] one obtain unique set $\Im^{\prime}=\left\{g_{k}\right\}_{k \in \mathbb{N}}$ such that $\forall k\left[g_{k} \in\left\{g_{n, k}\right\}_{n \in \mathbb{N}}\right]$.Finally one obtain a set $\Im$ from a set $\Im^{\prime}$ by axiom schema of replacement [10]. Thus one can define a $\mathbf{T h}^{\#}$-set $\Re_{c} \varsubsetneqq \Im$ :

$$
\forall x\left[x \in \Re_{c} \leftrightarrow(x \in \Im) \wedge \mathbf{P r}_{\mathbf{T h} \#}\left([x \notin x]^{c}\right)\right] .
$$

Proposition 2.3. Any collection $\Theta_{k}=g\left(\Psi_{k}\right), k=1,2, .$. is a $\mathbf{T h}^{\#}$-set.

Proof. We define $g_{n, k}=g\left(\Psi_{n, k}\left(x_{k}\right)\right)=\left[\Psi_{n, k}\left(x_{k}\right)\right]^{c}, v_{k}=\left[x_{k}\right]^{c}$. Therefore $g_{n, k}=g\left(\Psi_{n, k}\left(x_{k}\right)\right) \leftrightarrow$ $\operatorname{Fr}\left(g_{n, k}, v_{k}\right)$ (see [9]). Let us define now predicate $\Pi\left(g_{n, k}, v_{k}\right)$

$$
\left\{\begin{array}{c}
\Pi\left(g_{n, k}, v_{k}\right) \leftrightarrow \operatorname{Pr}_{\mathbf{T h}}\left(\left[\exists ! x_{k}\left[\Psi_{1, k}\left(x_{1}\right)\right]\right]^{c}\right) \wedge \\
\wedge \exists ! x_{k}\left(v_{k}=\left[x_{k}\right]^{c}\right)\left[\forall n(n \in \mathbb{N})\left[\operatorname{Pr}_{\mathbf{T h}}\left(\left[\left[\Psi_{1, k}\left(x_{k}\right)\right]\right]^{c}\right) \leftrightarrow \operatorname{Pr}_{\mathbf{T h}}\left(\mathbf{F r}\left(g_{n, k}, v_{k}\right)\right)\right]\right]
\end{array}\right.
$$


We define now a set $\Theta_{k}$ such that

$$
\left\{\begin{array}{c}
\Theta_{k}=\Theta_{k}^{\prime} \cup\left\{g_{k}\right\} \\
\forall n(n \in \mathbb{N})\left[g_{n, k} \in \Theta_{k}^{\prime} \leftrightarrow \Pi\left(g_{n, k}, v_{k}\right)\right]
\end{array}\right.
$$

But obviously definitions (2.19) and (2.25) is equivalent by Proposition 2.1.

Proposition 2.4. (i) $\mathbf{T h} \mathbf{h}^{\#} \vdash \exists \Re_{c}$, (ii) $\Re_{c}$ is a countable $\mathbf{T} \mathbf{h}^{\#}$-set.

Proof.(i) Statement $\mathbf{T} \mathbf{h}^{\#} \vdash \exists \Re_{c}$ follows immediately by using statement $\exists \Im$ and axiom schema of separation [4]. (ii) follows immediately from countability of a set $\Im$.

Proposition 2.5. A set $\Re_{c}$ is inconsistent.

Proof.From formla (2.18) one obtain

$$
\mathbf{T h}^{\#} \vdash \Re_{c} \in \Re_{c} \leftrightarrow \mathbf{P r}_{\mathbf{T h} \#}\left(\left[\Re_{c} \notin \Re_{c}\right]^{c}\right) .
$$

From formula (2.21) and Proposition 2.1 one obtain

$$
\mathbf{T h}^{\#} \vdash \Re_{c} \in \Re_{c} \leftrightarrow \Re_{c} \notin \Re_{c}
$$

and therefore

$$
\mathbf{T h}^{\#} \vdash\left(\Re_{c} \in \Re_{c}\right) \wedge\left(\Re_{c} \notin \Re_{c}\right) .
$$

But this is a contradiction.

Proposition 2.6. Assume that (i) Con (Th) and (ii ) Th has an nonstandard model $M_{N s t}^{\mathbf{T h}}$. Then theory $\mathbf{T h}$ can be extended to a maximally consistent nice theory $\mathbf{T h}^{\#} \triangleq \mathbf{T h}^{\#}\left[M_{\mathbf{N s t}}^{\mathbf{T h}}\right]$.

Proof. Let $\Phi_{1} \ldots \Phi_{i} \ldots$ be an enumeration of all wff's of the theory Th (this can be achieved if the set of propositional variables can be enumerated). Define a chain $\wp=\left\{\mathbf{T h}_{i} \mid i \in \mathbb{N}\right\}, \mathbf{T} \mathbf{h}_{1}=\mathbf{T h}$ of consistent theories inductively as follows: assume that theory $\mathbf{T} \mathbf{h}_{i}$ is defined. (i) Suppose that a statement (2.24) is satisfied

$$
\mathbf{T h}_{i} \vdash \mathbf{P r}_{\mathbf{T h}_{i}}\left(\left[\Phi_{i}\right]^{c}\right) \text { and }\left[\mathbf{T h}_{i} \nvdash \Phi_{i}\right] \wedge\left[M_{N s t}^{\mathbf{T h}} \models \Phi_{i}\right] .
$$

Then we define a theory $\mathbf{T} \mathbf{h}_{i+1}$ as follows $\mathbf{T} \mathbf{h}_{i+1} \triangleq \mathbf{T} \mathbf{h}_{i} \cup\left\{\Phi_{i}\right\}$.Using Lemma 2.1 we will rewrite the condition (2.24) symbolically as follows

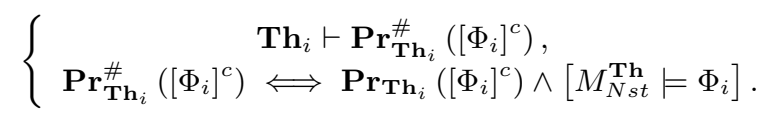

(ii) Suppose that a statement (2.26) is satisfied

$$
\mathbf{T h}_{i} \vdash \mathbf{P r}_{\mathbf{T h}_{i}}\left(\left[\neg \Phi_{i}\right]^{c}\right) \text { and }\left[\mathbf{T h}_{i} \nvdash \neg \Phi_{i}\right] \wedge\left[M_{N s t}^{\mathbf{T h}} \models \neg \Phi_{i}\right] .
$$

Then we define theory $\mathbf{T} \mathbf{h}_{i+1}$ as follows: $\mathbf{T} \mathbf{h}_{i+1} \triangleq \mathbf{T} \mathbf{h}_{i} \cup\left\{\neg \Phi_{i}\right\}$. Using Lemma 2.2 we will rewrite the condition (2.26) symbolically as follows

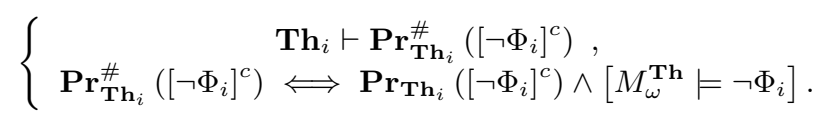

(iii) Suppose that a statement (2.28) is satisfied

$$
\mathbf{T h}_{i} \vdash \mathbf{P r}_{\mathbf{T h}_{i}}\left(\left[\Phi_{i}\right]^{c}\right) \text { and } \mathbf{T h}_{i} \vdash \mathbf{P r}_{\mathbf{T h}}\left(\left[\Phi_{i}\right]^{c}\right) \Longrightarrow \Phi_{i}
$$


We will rewrite the condition (2.28) symbolically as follows

$$
\left\{\begin{array}{c}
\mathbf{T h}_{i} \vdash \mathbf{P r}_{\mathbf{T h}_{i}}^{*}\left(\left[\Phi_{i}\right]^{c}\right), \\
\mathbf{P r}_{\mathbf{T h}_{i}}^{*}\left(\left[\Phi_{i}\right]^{c}\right) \Longleftrightarrow \mathbf{P r}_{\mathbf{T h}_{i}}\left(\left[\Phi_{i}\right]^{c}\right) \wedge\left[\mathbf{P r}_{\mathbf{T h}_{i}}\left(\left[\Phi_{i}\right]^{c}\right) \Longrightarrow \Phi_{i}\right]
\end{array}\right.
$$

Then we define a theory $\mathbf{T} \mathbf{h}_{i+1}$ as follows: $\mathbf{T} \mathbf{h}_{i+1} \triangleq \mathbf{T} \mathbf{h}_{i}$.

(iv) Suppose that a statement (2.30) is satisfied

$$
\mathbf{T h}_{i} \vdash \mathbf{P r}_{\mathbf{T h}_{i}}\left(\left[\neg \Phi_{i}\right]^{c}\right) \text { and } \mathbf{T h}_{i} \vdash \mathbf{P r}_{\mathbf{T h}_{i}}\left(\left[\neg \Phi_{i}\right]^{c}\right) \Longrightarrow \neg \Phi_{i} .
$$

We will rewrite the condition (2.30) symbolically as follows

$$
\left\{\begin{array}{c}
\operatorname{Th}_{i} \vdash \mathbf{P r}_{\mathbf{T h}_{i}}^{*}\left(\left[\Phi_{i}\right]^{c}\right), \\
\operatorname{Pr}_{\mathbf{T h}_{i}}^{*}\left(\left[\neg \Phi_{i}\right]^{c}\right) \Longleftrightarrow \operatorname{Pr}_{\mathbf{T h}_{i}}\left(\left[\neg \Phi_{i}\right]^{c}\right) \wedge\left[\mathbf{P r}_{\mathbf{T h}_{i}}\left(\left[\neg \Phi_{i}\right]^{c}\right) \Longrightarrow \neg \Phi_{i}\right]
\end{array}\right.
$$

Then we define a theory $\mathbf{T} \mathbf{h}_{i+1}$ as follows: $\mathbf{T} \mathbf{h}_{i+1} \triangleq \mathbf{T} \mathbf{h}_{i}$. We define now a theory $\mathbf{T} \mathbf{h}^{\#}$ as follows:

$$
\mathbf{T h}^{\#} \triangleq \bigcup_{i \in \mathbb{N}} \mathbf{T h}_{i}
$$

First, notice that each $\mathbf{T} \mathbf{h}_{i}$ is consistent. This is done by induction on $i$ and by Lemmas 2.1-2.2. By assumption, the case is true when $i=1$.Now, suppose $\mathbf{T} \mathbf{h}_{i}$ is consistent. Then its deductive closure $\operatorname{Ded}\left(\mathbf{T h}_{i}\right)$ is also consistent. If a statement (2.28) is satisfied,i.e. $\mathbf{T h} \vdash \mathbf{P r}_{\mathbf{T h}}\left(\left[\Phi_{i}\right]^{c}\right)$ and $\mathbf{T h} \vdash \Phi_{i}$, then clearly $\mathbf{T} \mathbf{h}_{i+1} \triangleq \mathbf{T} \mathbf{h}_{i} \cup\left\{\Phi_{i}\right\}$ is consistent since it is a subset of closure $\operatorname{Ded}\left(\mathbf{T} \mathbf{h}_{i}\right)$.If a statement (2.30) is satisfied,i.e. $\mathbf{T h} \vdash \mathbf{P r}_{\mathbf{T h}}\left(\left[\neg \Phi_{i}\right]^{c}\right)$ and $\mathbf{T h} \vdash \neg \Phi_{i}$, then clearly $\mathbf{T h}_{i+1} \triangleq \mathbf{T h}_{i} \cup\left\{\neg \Phi_{i}\right\}$ is consistent since it is a subset of closure $\mathbf{D e d}\left(\mathbf{T h}_{i}\right)$. Otherwise:(i) if a statement (2.24) is satisfied,i.e. $\mathbf{T h} \vdash \mathbf{P r}_{\mathbf{T h}}\left(\left[\Phi_{i}\right]^{c}\right)$ and $\left[\mathbf{T h}_{i} \nvdash \Phi_{i}\right] \wedge\left[M_{\omega}^{\mathbf{T h}} \models \Phi_{i}\right]$ then clearly $\mathbf{T h}_{i+1} \triangleq \mathbf{T h}_{i} \cup\left\{\Phi_{i}\right\}$ is consistent by Lemma 2.1 and by one of the standard properties of consistency: $\Delta \cup\{A\}$ is consistent iff $\Delta \nvdash \neg A$; (ii) if a statement (2.26) is satisfied,i.e. $\mathbf{T h}_{i} \vdash \mathbf{P r}_{\mathbf{T h}}\left(\left[\neg \Phi_{i}\right]^{c}\right)$ and $\left[\mathbf{T h}_{i} \nvdash \neg \Phi_{i}\right] \wedge\left[M_{\omega}^{\mathbf{T h}} \models \neg \Phi_{i}\right]$ then clearly $\mathbf{T h}_{i+1} \triangleq \mathbf{T h}_{i} \cup\left\{\neg \Phi_{i}\right\}$ is consistent by Lemma 2.2 and by one of the standard properties of consistency: $\Delta \cup\{\neg A\}$ is consistent iff $\Delta \nvdash A$.Next, notice Ded $\left(\mathbf{T h} \mathbf{h}^{\#}\right)$ is maximally consistent nice extension of the Ded ( $\mathbf{T h})$.Ded $\left(\mathbf{T h} \mathbf{h}^{\#}\right)$ is consistent because, by the standard Lemma 2.3 belov, it is the union of a chain of consistent sets. To see that Ded $\left(\mathbf{T h} \mathbf{h}^{\#}\right)$ is maximal, pick any wff $\Phi$. Then $\Phi$ is some $\Phi_{i}$ in the enumerated list of all wff's. Therefore for any $\Phi$ such that $\mathbf{T h}_{i} \vdash \mathbf{P r}_{\mathbf{T h}}\left([\Phi]^{c}\right)$ or $\mathbf{T h}_{i} \vdash \mathbf{P r}_{\mathbf{T h}}\left([\neg \Phi]^{c}\right)$, either $\Phi \in \mathbf{T} \mathbf{h}^{\#}$ or $\neg \Phi \in \mathbf{T} \mathbf{h}^{\#}$. Since Ded $\left(\mathbf{T} \mathbf{h}_{i+1}\right) \subseteq \operatorname{Ded}\left(\mathbf{T h} \mathbf{h}^{\#}\right)$, we have $\Phi \in \operatorname{Ded}\left(\mathbf{T h}{ }^{\#}\right)$ or $\neg \Phi \in \operatorname{Ded}\left(\mathbf{T h}{ }^{\#}\right)$, which implies that $\mathbf{D e d}\left(\mathbf{T} \mathbf{h}^{\#}\right)$ is maximally consistent nice extension of the Ded (Th).

Definition 2.7. We define now predicate $\mathbf{P r}_{\mathbf{T h}} \#\left(\left[\Phi_{i}\right]^{c}\right)$ asserting provability in $\mathbf{T h}{ }^{\#}$ :

$$
\left\{\begin{aligned}
\mathbf{P r}_{\mathbf{T h}} \#\left(\left[\Phi_{i}\right]^{c}\right) & \Longleftrightarrow\left[\mathbf{P r}_{\mathbf{T h}_{i}}^{\#}\left(\left[\Phi_{i}\right]^{c}\right)\right] \vee\left[\mathbf{P r}_{\mathbf{T h}}^{*}\left(\left[\Phi_{i}\right]^{c}\right)\right] \\
\mathbf{P r}_{\mathbf{T h} \#}\left(\left[\neg \Phi_{i}\right]^{c}\right) & \Longleftrightarrow\left[\mathbf{P r}_{\mathbf{T} \mathbf{h}_{i}}^{\#}\left(\left[\neg \Phi_{i}\right]^{c}\right)\right] \vee\left[\mathbf{P r}_{\mathbf{T} \mathbf{h}_{i}}^{*}\left(\left[\neg \Phi_{i}\right]^{c}\right)\right] .
\end{aligned}\right.
$$

Definition 2.8. Let $\Psi=\Psi(x)$ be one-place open wff such that the conditions:

(*) $\mathbf{T h} \vdash \exists ! x_{\Psi}\left[\Psi\left(x_{\Psi}\right)\right]$ or

$(* *) \mathbf{T h} \vdash \mathbf{P r}_{\mathbf{T h}}\left(\left[\exists ! x_{\Psi}\left[\Psi\left(x_{\Psi}\right)\right]\right]^{c}\right)$ and $M_{N s t}^{\mathbf{T h}} \models \exists ! x_{\Psi}\left[\Psi\left(x_{\Psi}\right)\right]$ is satisfied.

Then we said that, a set $y$ is a $\mathbf{T} \mathbf{h}^{\#}$-set iff there is exist one-place open wff $\Psi(x)$ such that $y=x_{\Psi}$. We write $y[\mathbf{T h} \#]$ iff $y$ is a $\mathbf{T h}^{\#}$-set.

Remark 2.4. Note that $[(*) \vee(* *)] \Longrightarrow \mathbf{T h}^{\#} \vdash \exists ! x_{\Psi}\left[\Psi\left(x_{\Psi}\right)\right]$. 
Remark 2.5. Note that $y\left[\mathbf{T h}^{\#}\right] \Longleftrightarrow \exists \Psi\left[\left(y=x_{\Psi}\right) \wedge \mathbf{P r}_{\mathbf{T h}} \#\left(\left[\exists ! x_{\Psi}\left[\Psi\left(x_{\Psi}\right)\right]\right]^{c}\right)\right]$

Definition 2.9. Let $\Im$ be a collection such that : $\forall x\left[x \in \Im \leftrightarrow x\right.$ is a $\mathbf{T h}^{\#}$-set $]$.

Proposition 2.7. Collection $\Im$ is a $\mathbf{T h}{ }^{\#}$-set.

Proof. Let us consider an one-place open wff $\Psi(x)$ such that conditions $(*)$ or $(* *)$ is satisfied, i.e. $\mathbf{T} \mathbf{h}^{\#} \vdash \exists ! x_{\Psi}\left[\Psi\left(x_{\Psi}\right)\right]$. We note that there exists countable collection $\Psi$ of the one-place open wff's

$$
\begin{aligned}
& \Psi=\left\{\Psi_{n}(x)\right\}_{n \in \mathbb{N}} \text { such that: (i) } \Psi(x) \in_{\Psi} \text { and (ii) }
\end{aligned}
$$

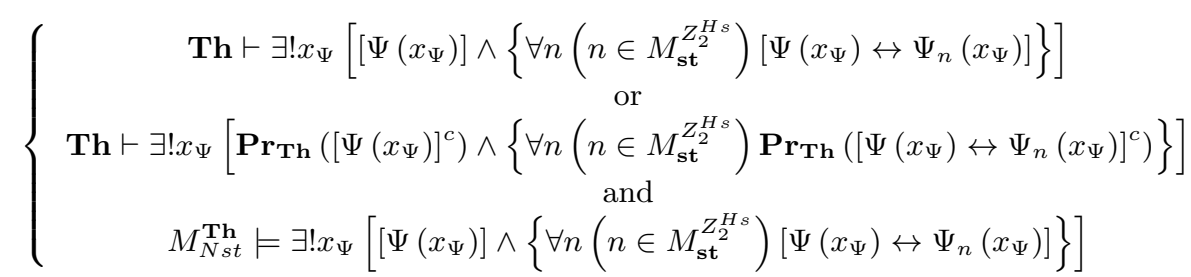

or of the equivalent form

$$
\left\{\begin{aligned}
\mathbf{T h} \vdash \exists ! x_{1}\left[[ \Psi _ { 1 } ( x _ { 1 } ) ] \wedge \left\{\begin{array}{rl}
\forall n\left(n \in M_{\mathbf{s t}}^{Z_{2}^{H s}}\right) \\
\text { or }
\end{array}\right.\right. & {\left.\left.\left[\Psi_{1}\left(x_{1}\right) \leftrightarrow \Psi_{n, 1}\left(x_{1}\right)\right]\right\}\right] } \\
\mathbf{T h} \vdash \exists ! x_{\Psi}\left[\mathbf{P r}_{\mathbf{T h}}\left(\left[\Psi\left(x_{1}\right)\right]^{c}\right)\right. & \wedge\left\{\begin{array}{c}
\left.\left.\forall n\left(n \in M_{\mathbf{s t}}^{Z_{2}^{H s}}\right) \mathbf{P r}_{\mathbf{T h}}\left(\left[\Psi\left(x_{1}\right) \leftrightarrow \Psi_{n}\left(x_{1}\right)\right]^{c}\right)\right\}\right] \\
\text { and }
\end{array}\right. \\
M_{N s t}^{\mathbf{T h}} \models \exists ! x_{\Psi}\left[\left[\Psi\left(x_{1}\right)\right]\right. & \left.\wedge\left\{\forall n\left(n \in M_{\mathbf{s t}}^{Z_{2}^{H s}}\right)\left[\Psi\left(x_{1}\right) \leftrightarrow \Psi_{n}\left(x_{1}\right)\right]\right\}\right]
\end{aligned}\right.
$$

where we set $\Psi(x)=\Psi_{1}\left(x_{1}\right), \Psi_{n}\left(x_{1}\right)=\Psi_{n, 1}\left(x_{1}\right)$ and $x_{\Psi}=x_{1}$. We note that any collection $\Psi_{k}=\left\{\Psi_{n, k}(x)\right\}_{n \in \mathbb{N}}, k=1,2, \ldots$ such above defines an unique set $x_{\Psi_{k}}$, i.e. $\quad \Psi_{k_{1}} \bigcap_{\Psi_{k_{2}}}=\varnothing$ iff $x_{\Psi_{k_{1}}} \neq x_{\Psi_{k_{2}}}$. We note that collections $\Psi_{k}, k=1,2, .$. is no part of the $Z F C_{2}^{H s}$,i.e. collection $\Psi_{k}$ there is no set in sense of $Z F C_{2}^{H s}$. However that is no problem, because by using Gödel numbering one can to replace any collection $\Psi_{k}, k=1,2, .$. by collection $\Theta_{k}=g\left(\Psi_{k}\right)$ of the corresponding Gödel numbers such that

$$
\Theta_{k}=g\left(\Psi_{k}\right)=\left\{g\left(\Psi_{n, k}\left(x_{k}\right)\right)\right\}_{n \in \mathbb{N}}, k=1,2, \ldots .
$$

It is easy to prove that any collection $\Theta_{k}=g\left(\Psi_{k}\right), k=1,2, .$. is a $\mathbf{T} \mathbf{h}^{\#}$-set. This is done by Gödel encoding [8],[9] of the statament (2.19) by Proposition 2.6 and by axiom schema of separation [4]. Let $g_{n, k}=g\left(\Psi_{n, k}\left(x_{k}\right)\right), k=1,2, .$. be a Gödel number of the wff $\Psi_{n, k}\left(x_{k}\right)$. Therefore $g(k)=$ $\left\{g_{n, k}\right\}_{n \in \mathbb{N}}$, where we set ${ }_{k}=\Psi_{k}, k=1,2, .$. and

$$
\forall k_{1} \forall k_{2}\left[\left\{g_{n, k_{1}}\right\}_{n \in \mathbb{N}} \cap\left\{g_{n, k_{2}}\right\}_{n \in \mathbb{N}}=\varnothing \leftrightarrow x_{k_{1}} \neq x_{k_{2}}\right]
$$

Let $\left\{\left\{g_{n, k}\right\}_{n \in \mathbb{N}}\right\}_{k \in \mathbb{N}}$ be a family of the all sets $\left\{g_{n, k}\right\}_{n \in \mathbb{N}}$. By axiom of choice [10] one obtain unique set $\Im^{\prime}=\left\{g_{k}\right\}_{k \in \mathbb{N}}$ such that $\forall k\left[g_{k} \in\left\{g_{n, k}\right\}_{n \in \mathbb{N}}\right]$. Finallyoneobtainaset $\Im$ from a set $\Im^{\prime}$ by axiom schema of replacement [10]. Thus one can define a $\mathbf{T h}^{\#}$-set $\Re_{c} \varsubsetneqq \Im$ :

$$
\forall x\left[x \in \Re_{c} \leftrightarrow(x \in \Im) \wedge \mathbf{P r}_{\mathbf{T h} \#}\left([x \notin x]^{c}\right)\right]
$$


Proposition 2.8. Any collection $\Theta_{k}=g\left(\Psi_{k}\right), k=1,2, .$. is a $\mathbf{T h}^{\#}$-set.

Proof. We define $g_{n, k}=g\left(\Psi_{n, k}\left(x_{k}\right)\right)=\left[\Psi_{n, k}\left(x_{k}\right)\right]^{c}, v_{k}=\left[x_{k}\right]^{c}$.

Therefore $g_{n, k}=g\left(\Psi_{n, k}\left(x_{k}\right)\right) \leftrightarrow \mathbf{F r}\left(g_{n, k}, v_{k}\right)$ (see [9]). Let us define now predicate $\Pi\left(g_{n, k}, v_{k}\right)$

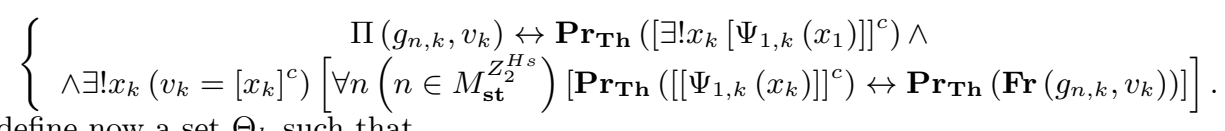

We define now a set $\Theta_{k}$ such that

$$
\left\{\begin{array}{c}
\Theta_{k}=\Theta_{k}^{\prime} \cup\left\{g_{k}\right\} \\
\forall n(n \in \mathbb{N})\left[g_{n, k} \in \Theta_{k}^{\prime} \leftrightarrow \Pi\left(g_{n, k}, v_{k}\right)\right]
\end{array}\right.
$$

But obviously definitions (2.39) and (2.40) is equivalent by Proposition 2.6.

Proposition 2.9. (i) $\mathbf{T h}^{\#} \vdash \exists \Re_{c}$,(ii) $\Re_{c}$ is a countable $\mathbf{T h}^{\#}$-set.

Proof.(i) Statement $\mathbf{T h}^{\#} \vdash \exists \Re_{c}$ follows immediately by using statement $\exists \Im$ and axiom schema of separation [10]. (ii) follows immediately from countability of a set $\Im$.

Proposition 2.10. A set $\Re_{c}$ is inconsistent.

Proof.From formla (2.18) one obtain

$$
\mathbf{T h}^{\#} \vdash \Re_{c} \in \Re_{c} \leftrightarrow \mathbf{P r}_{\mathbf{T h} \#}\left(\left[\Re_{c} \notin \Re_{c}\right]^{c}\right) .
$$

From formula (2.41) and Proposition 2.6 one obtain

$$
\mathbf{T h}^{\#} \vdash \Re_{c} \in \Re_{c} \leftrightarrow \Re_{c} \notin \Re_{c}
$$

and therefore

$$
\mathbf{T h}^{\#} \vdash\left(\Re_{c} \in \Re_{c}\right) \wedge\left(\Re_{c} \notin \Re_{c}\right)
$$

But this is a contradiction.

\section{Derivation of the Inconsistent Countable Set in $Z F C_{2}$ with the Full Semantics}

Let $\mathbf{T h}$ be an second order theory with the full second order semantics. We assume now that: (i) Th contains $Z F C_{2}^{f s s}$,(ii) Th has no any model.

Definition 3.1. Using formula (2.3) one can define predicate $\mathbf{P r}_{\mathbf{T h}}^{\omega}(y)$ really asserting provability in $Z F C_{2}^{f s s}$

$$
\operatorname{Pr}_{\mathbf{T h}}^{\omega}(y) \leftrightarrow \exists x\left(x \in M_{\omega}^{Z_{2}}\right) \operatorname{Prov}_{\mathbf{T h}}(x, y),
$$

Theorem 3.1.[11]. (Löb's Theorem for $Z F C_{2}$ ) Let $\Phi$ be any closed formula with code $y=[\Phi]^{c} \in M_{\omega}^{Z_{2}}$, then $\mathbf{T h} \vdash \mathbf{P r}_{\mathbf{T h}}^{\omega}\left([\Phi]^{c}\right)$ implies $\mathbf{T h} \vdash \Phi$ (see [12] Theorem 5.1).

Proof. Assume that

(\#) $\mathbf{T h} \vdash \mathbf{P r}_{\mathbf{T h}}^{\omega}\left([\Phi]^{c}\right)$. 
Note that

(1) $\mathbf{T h} \nvdash \neg \Phi$. Otherwise one obtain $\mathbf{T h} \vdash \mathbf{P r}_{\mathbf{T h}}^{\omega}\left([\neg \Phi]^{c}\right) \wedge \mathbf{P r}_{\mathbf{T h}}^{\omega}\left([\Phi]^{c}\right)$, but this is a contradiction.

(2) Assume now that (2.i) $\mathbf{T h} \vdash \mathbf{P r}_{\mathbf{T h}}^{\omega}\left([\Phi]^{c}\right)$ and (2.ii) $\mathbf{T h} \nvdash \Phi$.

From (1) and (2.ii) follows that

(3) $\mathbf{T h} \nvdash \neg \Phi$ and $\mathbf{T h} \nvdash \Phi$.

Let $\mathbf{T} \mathbf{h}_{\neg \Phi}$ be a theory

(4) $\mathbf{T h}_{\neg \Phi} \triangleq \mathbf{T h} \cup\{\neg \Phi\}$.From (3) follows that

(5) $\operatorname{Con}\left(\mathbf{T h}_{\neg \Phi}\right)$.

From (4) and (5) follows that

(6) $\mathbf{T h}_{\neg \Phi} \vdash \mathbf{P r}_{\mathbf{T h}_{\neg \Phi}}^{\omega}\left([\neg \Phi]^{c}\right)$.

From (4) and (\#) follows that

(7) $\mathbf{T h}_{\neg \Phi} \vdash \mathbf{P r}_{\mathbf{T h}}^{\omega} \mathbf{h}_{\neg \Phi}\left([\Phi]^{c}\right)$.

From (6) and (7) follows that

(8) $\mathbf{T h}_{\neg \Phi} \vdash \mathbf{P r}_{\mathbf{T h}}^{\omega} \mathbf{h}_{\neg \Phi}\left([\Phi]^{c}\right) \wedge \mathbf{P r}_{\mathbf{T h}}^{\omega} \mathbf{h}_{\neg \Phi}\left([\neg \Phi]^{c}\right)$, but this is a contradiction.

Definition 3.2. Let $\Psi=\Psi(x)$ be one-place open wff such that the conditions:

$(*) \mathbf{T h} \vdash \exists ! x_{\Psi}\left[\Psi\left(x_{\Psi}\right)\right]$ or

$(* *) \mathbf{T h} \vdash \mathbf{P r}_{\mathbf{T h}}^{\omega}\left(\left[\exists ! x_{\Psi}\left[\Psi\left(x_{\Psi}\right)\right]\right]^{c}\right)$ is satisfied.

Then we said that, a set $y$ is a Th-set iff there is exist one-place open wff $\Psi(x)$ such that $y=x_{\Psi}$. We write $y[\mathbf{T h}]$ iff $y$ is a $\mathbf{T h}$-set.

Remark 3.1. Note that $[(*) \vee(* *)] \Longrightarrow \mathbf{T h} \vdash \exists ! x_{\Psi}\left[\Psi\left(x_{\Psi}\right)\right]$.

Remark 3.2. Note that $y[\mathbf{T h}] \Longleftrightarrow \exists \Psi\left[\left(y=x_{\Psi}\right) \wedge \operatorname{Pr}_{\mathbf{T h}}^{\omega}\left(\left[\exists ! x_{\Psi}\left[\Psi\left(x_{\Psi}\right)\right]\right]^{c}\right)\right]$

Definition 3.3. Let $\Im$ be a collection such that : $\forall x[x \in \Im \leftrightarrow x$ is a Th-set $]$.

Proposition 3.2. Collection $\Im$ is a Th-set.

Definition 3.4. We define now a Th-set $\Re_{c} \varsubsetneqq \Im$ :

$$
\forall x\left[x \in \Re_{c} \leftrightarrow(x \in \Im) \wedge \operatorname{Pr}_{\mathbf{T h}}^{\omega}\left([x \notin x]^{c}\right)\right] .
$$

Proposition 3.3. (i) $\mathbf{T h} \vdash \exists \Re_{c}$, (ii) $\Re_{c}$ is a countable $\mathbf{T h}$-set.

Proof.(i) Statement $\mathbf{T h} \vdash \exists \Re_{c}$ follows immediately by using statement $\exists \Im$ and axiom schema of separation [4]. (ii) follows immediately from countability of a set $\Im$.

Proposition 3.4. A set $\Re_{c}$ is inconsistent.

Proof.From formla (3.2) one obtain

$$
\mathbf{T h} \vdash \Re_{c} \in \Re_{c} \leftrightarrow \mathbf{P r}_{\mathbf{T h}}^{\omega}\left(\left[\Re_{c} \notin \Re_{c}\right]^{c}\right) .
$$

From formula (3.3) and definition 3.1 one obtain

$$
\mathbf{T h} \vdash \Re_{c} \in \Re_{c} \leftrightarrow \Re_{c} \notin \Re_{c}
$$

and therefore

$$
\mathbf{T h} \vdash\left(\Re_{c} \in \Re_{c}\right) \wedge\left(\Re_{c} \notin \Re_{c}\right) .
$$


But this is a contradiction.

Therefore finally we obtain:

Theorem 3.2. [12]. $\neg \operatorname{Con}\left(Z F C_{2}\right)$.

That is well known that under $Z F C$ it can be shown that $k$ is inaccessible if and only if $\left(V_{k}, \in\right)$ is a model of $Z F C_{2}$ [5],[11].Thus finally we obtain.

Theorem 3.3. [12]. $\neg \operatorname{Con}\left(Z F C+\left(V=H_{k}\right)\right)$.

\section{Conclusion}

In this paper we have proved that the second order $Z F C$ with the full second-order semantic is inconsistent,i.e. $\neg \operatorname{Con}\left(Z F C_{2}\right)$. Main result is: let $k$ be an inaccessible cardinal and $H_{k}$ is a set of all sets having hereditary size less then $k$, then $\neg \operatorname{Con}\left(Z F C+\left(V=H_{k}\right)\right)$. This result also was obtained in $[7],[11],[12]$ by using essentially another approach. For the first time this result has been declared to AMS in [13],[14]. An important applications in topology and homotopy theory are obtained in $[15],[16],[17]$.

\section{Acknowledgments}

A reviewers provided important clarifications.

\section{Competing Interests}

The author declares that no competing interests exist.

\section{References}

[1] Nelson E. Warning signs of a possible collapse of contemporary mathematics. Available: https://web.math.princeton.edu/ nelson/papers/warn.pdf

[2] Henkin L. Completeness in the theory of types. Journal of Symbolic Logic. 1950;15(2):81-91. DOI:10.2307/2266967. JSTOR 2266967.

[3] Rossberg M. First-order logic, second-order logic, and completeness. In V. Hendricks et al., eds. First-order logic revisited. Berlin: Logos-Verlag.

[4] Shapiro S. Foundations without foundationalism: A case for second-order logic. Oxford University Press. ISBN 0-19-825029-0.

[5] Rayo A, Uzquiano G. Toward a theory of second-order consequence. Notre Dame Journal of Formal Logic.1999;40(3).

[6] Bovykin A. On order-types of models of arithmetic. Ph.D. thesis University of Birmingham. 2000;109. 
[7] Foukzon J. Strong reflection principles and large cardinal axioms. Pure and Applied Mathematics Journal. 2(3);119-127.

Available:http://www.sciencepublishinggroup.com/j/pam

DOI: $10.1 \overline{1648 / j . p a m j .20130203 .12 .}$

[8] Smorynski C. Handbook of mathematical logic. Edited by J. Barwise. North-Holland Publishing Company; 1977.

[9] Mendelson E. Introduction to mathematical logic. June 1, 1997. ISBN-10: 0412808307. ISBN13: 978-0412808302.

[10] Takeuti G, Zaring WM. Introduction to axiomatic set theory. Springer-Verlag; 1971.

[11] Foukzon J. Generalized Lob's theorem. Strong reflection principles and large cardinal axioms. Consistency Results in Topology.

Available: http://arxiv.org/abs/1301.5340

[12] Foukzon J. Inconsistent Countable Set. Available: http://vixra.org/abs/1302.0048

[13] Foukzon J, Men'kova ER. Generalized Löb's Theorem. strong reflection principles and large cardinal axioms. Advances in Pure Mathematics. 2013;3(3).

[14] Foukzon J. Strong reflection principles and large cardinal axioms. Fall Southeastern Sectional Meeting University of Louisville, Louisville, KY October 5-6, 2013 (Saturday -Sunday) Meeting \#1092.

Available:http://www.ams.org/amsmtgs/2208_abstracts/1092-03-13.pdf

[15] Foukzon J. Consistency results in topology and homotopy theory. Pure and Applied Mathematics Journal. 2015;4(1-1):1-5. Published online October 29, 2014.

DOI: 10.11648/j.pamj.s.2015040101.11. ISSN: 2326-9790 (Print); ISSN: 2326-9812 (Online).

[16] Foukzon J. Generalized lob's theorem. Strong reflection. principles and large cardinal axioms. Consistency Results in Topology, IX Iberoamerican Conference on Topology and its Applications 24-27 June, Almeria, Spain. Book of abstracts. 2014;66.

[17] Foukzon J. Generalized lob's theorem. Strong reflection principles and large cardinal axioms. Consistency Results in Topology, International Conference on Topology and its Applications, July 3-7, Nafpaktos, Greece. Book of abstracts. 2014;81.

(C)2015 Foukzon; This is an Open Access article distributed under the terms of the Creative Commons Attribution License http://creativecommons.org/licenses/by/4.0, which permits unrestricted use, distribution, and reproduction in any medium, provided the original work is properly cited.

Peer-review history:

The peer review history for this paper can be accessed here (Please copy paste the total link in your browser address bar)

www.sciencedomain.org/review-history.php?iid=114683id=683aid=9622 